\title{
SPOKEN LANGUAGE SYSTEMS
}

\author{
PI: John Makhoul \\ Bolt Beranek \& Newman Inc., 10 Moulton St, Cambridge, MA 02138 \\ makhoul@bbn.com
}

The objective of this project is to develop a real-time spoken language system capable of understanding and responding to spoken English commands and queries for interactive human-machine applications, such as battle management, command and control, and training of personnel on complex tasks. The system will also include a capability to adapt to new speakers and a capability to detect when a user says a new word, and to allow the user to add the word to the system.

Work in this area requires the integration of three technologies: large-vocabulary continuous speech recognition, natural language understanding, and system integration. In our work at BBN, we have integrated our BYBLOS continuous speech recognition technology with a new unification-based natural language understanding component, resulting in an initial complete spoken language system, called HARC (Hear And Respond to Continuous speech).

The natural language knowledge sources in HARC use a Unification formalism for describing the syntax and semantics of English and a higher-order intensional logic for representing the meaning of an utterance. The system uses unification to enforce syntactic as well as semantic constraints, and provides for the incremental application of syntax and semantics. Advantages of this approach are that unproductive search paths are cut off more quickly, and any improvements in unification parsing (through better algorithms, special hardware, etc.) apply automatically to semantics as well as syntax. We have implemented unification semantics for our grammar rules in three task domains: battle management, personnel information retrieval, and airline travel information retrieval.

To help resolve ambiguities found in the semantics, we have interfaced the JANUS discourse module, developed under an earlier DARPA effort, to the HARC system. We also developed a method for rapid porting of the natural language component to new task domains using the Parlance Learner ${ }^{\mathrm{TM}}$ knowledge acquisition tool.

One important contribution has been the development of the N-best search strategy for integrating speech and natural language components. This method takes a spoken utterance and produces the $\mathbf{N}$ highest scoring sentences that match the input utterance within some threshold, based on a statistical language model. The natural language component then searches these $\mathrm{N}$ sentences for the highest scoring sentence for which the system can produce a semantic interpretation. One important feature of this $\mathrm{N}$-best integration strategy is that it provides a very clean interface between speech and natural language and, therefore, allows for greater sharing of resources among researchers in spoken language systems.

In this project, we have been instrumental in the design and collection of spoken language data for the purpose of objective system evaluation. We previously helped specify the DARPA Resource Management Corpus that is now in common use for speech recognition evaluation, and we provided a Word-Pair Grammar to be used with the corpus. More recently, we proposed a methodology for the collection and evaluation of spoken language data. We also developed and made available to the DARPA community a personnel database for use in spoken language evaluations, and a relational database language (ERL) in Common LISP to interface to the database. We have also provided software to aid in the collection of an appropriate corpus by Texas Instruments.

Recently, we developed what we believe to be the first successful method for the automatic detection of out-of-vocabulary words. This is an important problem for any realistic system with a large vocabulary, since the user is unlikely to be able to remember which words are in the vocabulary. Initial results show a $70 \%$ detection rate with only $1 \%$ false alarm.

We have now started the development of a real-time spoken language system using commercially available hardware. The first part of the effort will demonstrate $\mathrm{N}$-best recognition in real time. 